\title{
Searches for relativistic magnetic monopoles in IceCube
}

\author{
IceCube Collaboration
}

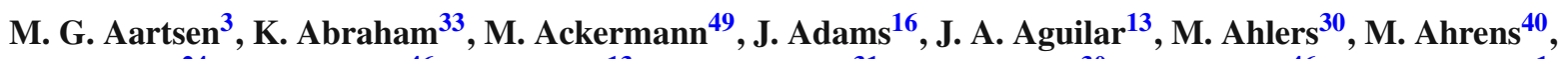
D. Altmann ${ }^{24}$, T. Anderson ${ }^{46}$, I. Ansseau ${ }^{13}$, M. Archinger ${ }^{31}$, C. Arguelles ${ }^{30}$, T. C. Arlen ${ }^{46}$, J. Auffenberg ${ }^{1}$, X. Bai ${ }^{38}$, S. W. Barwick ${ }^{27}$, V. Baum ${ }^{31}$, R. Bay ${ }^{8}$, J. J. Beatty ${ }^{18,19}$, J. Becker Tjus ${ }^{11}$, K.-H. Becker ${ }^{48}$, E. Beiser ${ }^{30}$, M. L. Benabderrahmane ${ }^{2}$, P. Berghaus ${ }^{49}$, D. Berley ${ }^{17}$, E. Bernardini ${ }^{49}$, A. Bernhard ${ }^{33}$, D. Z. Besson ${ }^{28}$, G. Binder ${ }^{8,9}$, D. Bindig ${ }^{48}$, M. Bissok ${ }^{1}$, E. Blaufuss ${ }^{17}$, J. Blumenthal ${ }^{1}$, D. J. Boersma ${ }^{47}$, C. Bohm ${ }^{40}$, M. Börner ${ }^{21}$, F. Bos ${ }^{11}$, D. Bose ${ }^{42}$, S. Böser ${ }^{31}$, O. Botner ${ }^{47}$, J. Braun ${ }^{30}$, L. Brayeur ${ }^{14}$, H.-P. Bretz ${ }^{49}$, N. Buzinsky ${ }^{23}$, J. Casey ${ }^{6}$, M. Casier ${ }^{14}$, E. Cheung ${ }^{17}$, D. Chirkin ${ }^{30}$, A. Christov ${ }^{25}$, K. Clark ${ }^{43}$, L. Classen ${ }^{24}$, S. Coenders ${ }^{33}$, D. F. Cowen ${ }^{45,46}$, A. H. Cruz Silva ${ }^{49}$, J. Daughhetee ${ }^{6}$, J. C. Davis ${ }^{18}$, M. Day ${ }^{30}$, J. P. A. M. de Andréé ${ }^{22}$, C. De Clercq $^{14}$, E. del Pino Rosendo ${ }^{31}$, H. Dembinski ${ }^{34}$, S. De Ridder ${ }^{26}$, P. Desiati ${ }^{30}$, K. D. de Vries ${ }^{14}$, G. de Wasseige ${ }^{14}$, M. de With ${ }^{10}$, T. DeYoung ${ }^{22}$, J. C. Díaz-Vélez ${ }^{30}$, V. di Lorenzo ${ }^{31}$, J. P. Dumm ${ }^{40}$, M. Dunkman ${ }^{46}$, B. Eberhardt ${ }^{31}$, T. Ehrhardt ${ }^{31}$, B. Eichmann ${ }^{11}$, S. Euler ${ }^{47}$, P. A. Evenson ${ }^{34}$, S. Fahey ${ }^{30}$, A. R. Fazely ${ }^{7}$, J. Feintzeig ${ }^{30}$, J. Felde ${ }^{17}$, K. Filimonov ${ }^{8}$, C. Finley ${ }^{40}$, T. Fischer-Wasels ${ }^{48}$, S. Flis ${ }^{40}$, C.-C. Fösig ${ }^{31}$, T. Fuchs ${ }^{21}$, T. K. Gaisser ${ }^{34}$, R. Gaior ${ }^{15}$, J. Gallagher ${ }^{29}$, L. Gerhardt ${ }^{8,9}$, K. Ghorbani ${ }^{30}$, D. Gier ${ }^{1}$, L. Gladstone ${ }^{30}$, M. Glagla ${ }^{1}$, T. Glüsenkamp ${ }^{49}$, A. Goldschmidt ${ }^{9}$, G. Golup ${ }^{14}$, J. G. Gonzalez ${ }^{34}$, D. Góra ${ }^{49}$, D. Grant ${ }^{23}$, Z. Griffith ${ }^{30}$, A. Groß ${ }^{33}$, C. Ha $^{8,9}$, C. Haack ${ }^{1}$, A. Haj Ismail ${ }^{26}$, A. Hallgren ${ }^{47}$, F. Halzen ${ }^{30}$, E. Hansen ${ }^{20}$, B. Hansmann ${ }^{1}$, K. Hanson ${ }^{30}$, D. Hebecker ${ }^{10}$, D. Heereman ${ }^{13}$, K. Helbing ${ }^{48}$, R. Hellauer ${ }^{17}$, S. Hickford ${ }^{48}$, J. Hignight ${ }^{22}$, G. C. Hill ${ }^{3}$, K. D. Hoffman ${ }^{17}$, R. Hoffmann ${ }^{48}$, K. Holzapfel ${ }^{33}$,

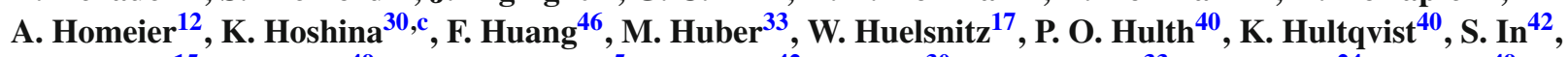

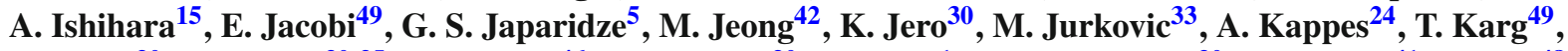
A. $\operatorname{Karle}^{30}$, M. Kauer ${ }^{30,35}$, A. Keivani ${ }^{46}$, J. L. Kelley ${ }^{30}$, J. Kemp ${ }^{1}$, A. Kheirandish ${ }^{30}$, J. Kiryluk ${ }^{41}$, J. Kläs ${ }^{48}$, S. R. Klein ${ }^{8,9}$, G. Kohnen ${ }^{32}$, R. Koirala ${ }^{34}$, H. Kolanoski ${ }^{10}$, R. Konietz ${ }^{1}$, L. Köpke ${ }^{31}$, C. Kopper ${ }^{23}$, S. Kopper ${ }^{48}$, D. J. Koskinen ${ }^{20}$, M. Kowalski ${ }^{10,49}$, K. Krings ${ }^{33}$, G. Kroll ${ }^{31}$, M. Kroll ${ }^{11}$, G. Krückl ${ }^{31}$, J. Kunnen ${ }^{14}$, N. Kurahashi ${ }^{37}$, T. Kuwabara ${ }^{15}$, M. Labare $^{26}$, J. L. Lanfranchi ${ }^{46}$, M. J. Larson ${ }^{20}$, M. Lesiak-Bzdak ${ }^{41}$, M. Leuermann ${ }^{1}$, J. Leuner ${ }^{1}$, L. Lu ${ }^{15}$, J. Lünemann ${ }^{14}$, J. Madsen ${ }^{39}$, G. Maggi ${ }^{14}$, K. B. M. Mahn ${ }^{22}$, M. Mandelartz ${ }^{11}$, R. Maruyama ${ }^{35}$,

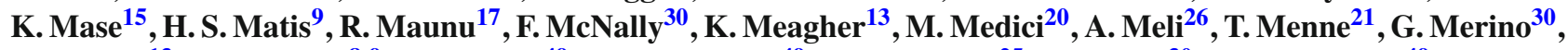
T. Meures $^{13}$, S. Miarecki ${ }^{8,9}$, E. Middell ${ }^{49}$, L. Mohrmann ${ }^{49}$, T. Montaruli ${ }^{25}$, R. Morse $^{30}$, R. Nahnhauer ${ }^{49}$, U. Naumann $^{48}$, G. Neer ${ }^{22}$, H. Niederhausen ${ }^{41}$, S. C. Nowicki ${ }^{23}$, D. R. Nygren ${ }^{9}$, A. Obertacke Pollmann ${ }^{48, a}$, A. Olivas ${ }^{17}$, A. Omairat ${ }^{48}$, A. O'Murchadha ${ }^{13}$, T. Palczewski ${ }^{44}$, H. Pandya ${ }^{34}$, D. V. Pankova ${ }^{46}$, L. Paul ${ }^{1}$, J. A. Pepper ${ }^{44}$, C. Pérez de los Heros ${ }^{47}$, C. Pfendner ${ }^{18}$, D. Pieloth ${ }^{21}$, E. Pinat ${ }^{13}$, J. Posselt ${ }^{48, b}$, P. B. Price ${ }^{8}$, G. T. Przybylski ${ }^{9}$, J. Pütz ${ }^{1}$, M. Quinnan ${ }^{46}$, C. Raab ${ }^{13}$, L. Rädel ${ }^{1}$, M. Rameez ${ }^{25}$, K. Rawlins ${ }^{4}$, R. Reimann ${ }^{1}$,

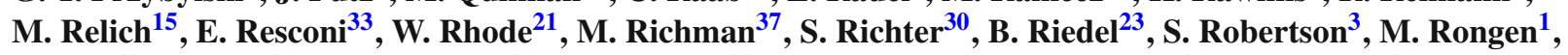
C. Rott $^{42}$, T. Ruhe ${ }^{21}$, D. Ryckbosch ${ }^{26}$, L. Sabbatini ${ }^{30}$, H.-G. Sander ${ }^{31}$, A. Sandrock ${ }^{21}$, J. Sandroos ${ }^{31}$, S. Sarkar ${ }^{20,36}$, K. Schatto ${ }^{31}$, F. Scheriau ${ }^{21}$, M. Schimp ${ }^{1}$, T. Schmidt ${ }^{17}$, M. Schmitz ${ }^{21}$, S. Schoenen ${ }^{1}$, S. Schöneberg ${ }^{11}$, A. Schönwald ${ }^{49}$, L. Schulte ${ }^{12}$, L. Schumacher ${ }^{1}$, D. Seckel ${ }^{34}$, S. Seunarine ${ }^{39}$, D. Soldin ${ }^{48}$, M. Song $^{17}$, G. M. Spiczak ${ }^{39}$, C. Spiering ${ }^{49}$, M. Stahlberg ${ }^{1}$, M. Stamatikos ${ }^{18, d}$, T. Stanev $^{34}$, A. Stasik ${ }^{49}$, A. Steuer ${ }^{31}$, T. Stezelberger ${ }^{9}$, R. G. Stokstad ${ }^{9}$, A. Stößl ${ }^{49}$, R. Ström ${ }^{47}$, N. L. Strotjohann ${ }^{49}$, G. W. Sullivan ${ }^{17}$, M. Sutherland ${ }^{18}$, H. Taavola ${ }^{47}$, I.

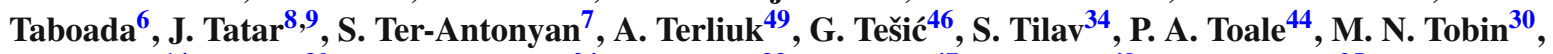

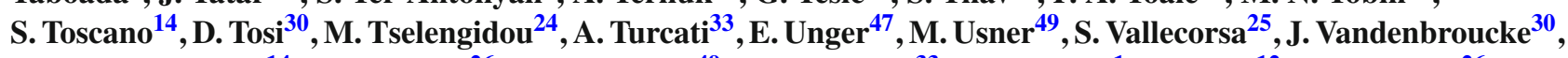
N. van Eijndhoven ${ }^{14}$, S. Vanheule ${ }^{26}$, J. van Santen ${ }^{49}$, J. Veenkamp ${ }^{33}$, M. Vehring ${ }^{1}$, M. Voge ${ }^{12}$, M. Vraeghe ${ }^{26}$, C. Walck $^{40}$, A. Wallace ${ }^{3}$, M. Wallraff ${ }^{1}$, N. Wandkowsky ${ }^{30}$, Ch. Weaver $^{23}$, C. Wendt $^{30}$, S. Westerhof $^{30}$, B. J. Whelan $^{3}$, K. Wiebe ${ }^{31}$, C. H. Wiebusch ${ }^{1}$, L. Wille ${ }^{30}$, D. R. Williams ${ }^{44}$, H. Wissing ${ }^{17}$, M. Wolf ${ }^{40}$, T. R. Wood ${ }^{23}$, K. Woschnagg $^{8}$,

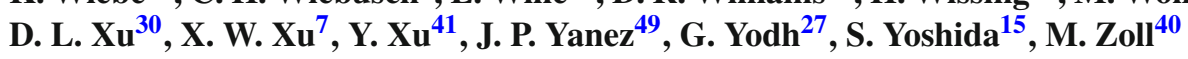

\footnotetext{
${ }^{1}$ III. Physikalisches Institut, RWTH Aachen University, 52056 Aachen, Germany

2 New York University Abu Dhabi, Abu Dhabi, United Arab Emirates

${ }^{3}$ Department of Physics, University of Adelaide, Adelaide 5005, Australia

${ }^{4}$ Department of Physics and Astronomy, University of Alaska Anchorage, 3211 Providence Dr., Anchorage, AK 99508, USA
} 
${ }^{5}$ CTSPS, Clark-Atlanta University, Atlanta, GA 30314, USA

${ }^{6}$ School of Physics and Center for Relativistic Astrophysics, Georgia Institute of Technology, Atlanta, GA 30332, USA

7 Department of Physics, Southern University, Baton Rouge, LA 70813, USA

${ }^{8}$ Department of Physics, University of California, Berkeley, CA 94720, USA

${ }^{9}$ Lawrence Berkeley National Laboratory, Berkeley, CA 94720, USA

${ }^{10}$ Institut für Physik, Humboldt-Universität zu Berlin, 12489 Berlin, Germany

${ }^{11}$ Fakultät für Physik \& Astronomie, Ruhr-Universität Bochum, 44780 Bochum, Germany

12 Physikalisches Institut, Universität Bonn, Nussallee 12, 53115 Bonn, Germany

${ }^{13}$ Université Libre de Bruxelles, Science Faculty CP230, 1050 Brussels, Belgium

14 Vrije Universiteit Brussel, Dienst ELEM, 1050 Brussels, Belgium

15 Department of Physics, Chiba University, Chiba 263-8522, Japan

${ }^{16}$ Department of Physics and Astronomy, University of Canterbury, Private Bag 4800, Christchurch, New Zealand

17 Department of Physics, University of Maryland, College Park, MD 20742, USA

${ }^{18}$ Department of Physics and Center for Cosmology and Astro-Particle Physics, Ohio State University, Columbus, OH 43210, USA

${ }^{19}$ Department of Astronomy, Ohio State University, Columbus, OH 43210, USA

${ }^{20}$ Niels Bohr Institute, University of Copenhagen, 2100 Copenhagen, Denmark

${ }^{21}$ Department of Physics, TU Dortmund University, 44221 Dortmund, Germany

22 Department of Physics and Astronomy, Michigan State University, East Lansing, MI 48824, USA

${ }^{23}$ Department of Physics, University of Alberta, Edmonton, Alberta, Canada T6G 2E1

${ }^{24}$ Erlangen Centre for Astroparticle Physics, Friedrich-Alexander-Universität Erlangen-Nürnberg, 91058 Erlangen, Germany

${ }^{25}$ Département de physique nucléaire et corpusculaire, Université de Genève, 1211 Geneva, Switzerland

${ }^{26}$ Department of Physics and Astronomy, University of Gent, 9000 Gent, Belgium

${ }^{27}$ Department of Physics and Astronomy, University of California, Irvine, CA 92697, USA

${ }^{28}$ Department of Physics and Astronomy, University of Kansas, Lawrence, KS 66045, USA

${ }^{29}$ Department of Astronomy, University of Wisconsin, Madison, WI 53706, USA

${ }^{30}$ Department of Physics and Wisconsin IceCube Particle Astrophysics Center, University of Wisconsin, Madison, WI 53706, USA

${ }^{31}$ Institute of Physics, University of Mainz, Staudinger Weg 7, 55099 Mainz, Germany

${ }^{32}$ Université de Mons, 7000 Mons, Belgium

33 Technische Universität München, 85748 Garching, Germany

${ }^{34}$ Department of Physics and Astronomy, Bartol Research Institute, University of Delaware, Newark, DE 19716, USA

35 Department of Physics, Yale University, New Haven, CT 06520, USA

${ }^{36}$ Department of Physics, University of Oxford, 1 Keble Road, Oxford OX1 3NP, UK

37 Department of Physics, Drexel University, 3141 Chestnut Street, Philadelphia, PA 19104, USA

38 Physics Department, South Dakota School of Mines and Technology, Rapid City, SD 57701, USA

${ }^{39}$ Department of Physics, University of Wisconsin, River Falls, WI 54022, USA

40 Department of Physics, Oskar Klein Centre, Stockholm University, 10691 Stockholm, Sweden

${ }^{41}$ Department of Physics and Astronomy, Stony Brook University, Stony Brook, NY 11794-3800, USA

42 Department of Physics, Sungkyunkwan University, Suwon 440-746, Korea

${ }^{43}$ Department of Physics, University of Toronto, Toronto, ON, Canada M5S 1A7

${ }^{44}$ Department of Physics and Astronomy, University of Alabama, Tuscaloosa AL 35487, USA

45 Department of Astronomy and Astrophysics, Pennsylvania State University, University Park, PA 16802, USA

46 Department of Physics, Pennsylvania State University, University Park, PA 16802, USA

47 Department of Physics and Astronomy, Uppsala University, Box 516, 75120 Uppsala, Sweden

48 Department of Physics, University of Wuppertal, 42119 Wuppertal, Germany

${ }^{49}$ DESY, 15735 Zeuthen, Germany

Received: 4 November 2015 / Accepted: 16 February 2016 / Published online: 10 March 2016

(C) The Author(s) 2016. This article is published with open access at Springerlink.com

\begin{abstract}
Various extensions of the Standard Model motivate the existence of stable magnetic monopoles that could have been created during an early high-energy epoch of the Universe. These primordial magnetic monopoles would be gradually accelerated by cosmic magnetic fields and could reach high velocities that make them visible in

\footnotetext{
a e-mail: anna.pollmann@uni-wuppertal.de

b e-mail: jposselt@icecube.wisc.edu

${ }^{c}$ Earthquake Research Institute, University of Tokyo, Bunkyo,

Tokyo 113-0032, Japan

d NASA Goddard Space Flight Center, Greenbelt, MD 20771, USA
}

Cherenkov detectors such as IceCube. Equivalently to electrically charged particles, magnetic monopoles produce direct and indirect Cherenkov light while traversing through matter at relativistic velocities. This paper describes searches for relativistic $(v \geq 0.76 c)$ and mildly relativistic $(v \geq 0.51 c)$ monopoles, each using one year of data taken in 2008/2009 and 2011/2012, respectively. No monopole candidate was detected. For a velocity above $0.51 c$ the monopole flux is constrained down to a level of $1.55 \times 10^{-18} \mathrm{~cm}^{-2} \mathrm{~s}^{-1} \mathrm{sr}^{-1}$. This is an improvement of almost two orders of magnitude over previous limits. 


\section{Introduction}

In Grand Unified Theories (GUTs) the existence of magnetic monopoles follows from general principles [1,2]. Such a theory is defined by a non-abelian gauge group that is spontaneously broken at a high energy to the Standard Model of particle physics [3]. The condition that the broken symmetry contains the electromagnetic gauge group $\mathrm{U}(1)_{\mathrm{EM}}$ is sufficient for the existence of magnetic monopoles [4]. Under these conditions the monopole is predicted to carry a magnetic charge $g$ governed by Dirac's quantization condition [5]

$g=n \cdot g_{D}=n \cdot \frac{e}{2 \alpha}$

where $n$ is an integer, $g_{D}$ is the elemental magnetic charge or Dirac charge, $\alpha$ is the fine structure constant, and $e$ is the elemental electric charge.

In a given GUT model the monopole mass can be estimated by the unification scale $\Lambda_{\mathrm{GUT}}$ and the corresponding value of the running coupling constant $\alpha_{\mathrm{GUT}}$ as $M c^{2} \gtrsim$ $\Lambda_{\mathrm{GUT}} / \alpha_{\mathrm{GUT}}$. Depending on details of the GUT model, the monopole mass can range from $10^{7}$ to $10^{17} \mathrm{GeV} / c^{2}[6,7]$. In any case, GUT monopoles are too heavy to be produced in any existing or foreseeable accelerator.

After production in the very early hot universe, their relic abundance is expected to have been exponentially diluted during inflation. However, monopoles associated with the breaking of intermediate scale gauge symmetries may have been produced in the late stages of inflation and reheating in some models $[8,9]$. There is thus no robust theoretical prediction of monopole parameters such as mass and flux, nevertheless an experimental detection of a monopole today would be of fundamental significance.

In this paper we present results for monopole searches with the IceCube Neutrino telescope covering a large velocity range. Due to the different light-emitting mechanisms at play, we present two analyses, each optimized according to their velocity range: highly relativistic monopoles with $v \geq 0.76 c$ and mildly relativistic monopoles with $v \geq 0.4 c$. The highly relativistic monopole analysis was performed with IceCube in its 40-string configuration while the mildly relativistic monopole analysis uses the complete 86-string detector.

The paper is organized as follows. In Sect. 2 we introduce the neutrino detector IceCube and describe in Sect. 3 the methods to detect magnetic monopoles with Cherenkov telescopes. We describe the simulation of magnetic monopoles in Sect. 4. The analyses for highly and mildly relativistic monopoles use different analysis schemes which are described in Sects. 5 and 6. The result of both analyses and an outlook is finally shown in Sects. 7-9.

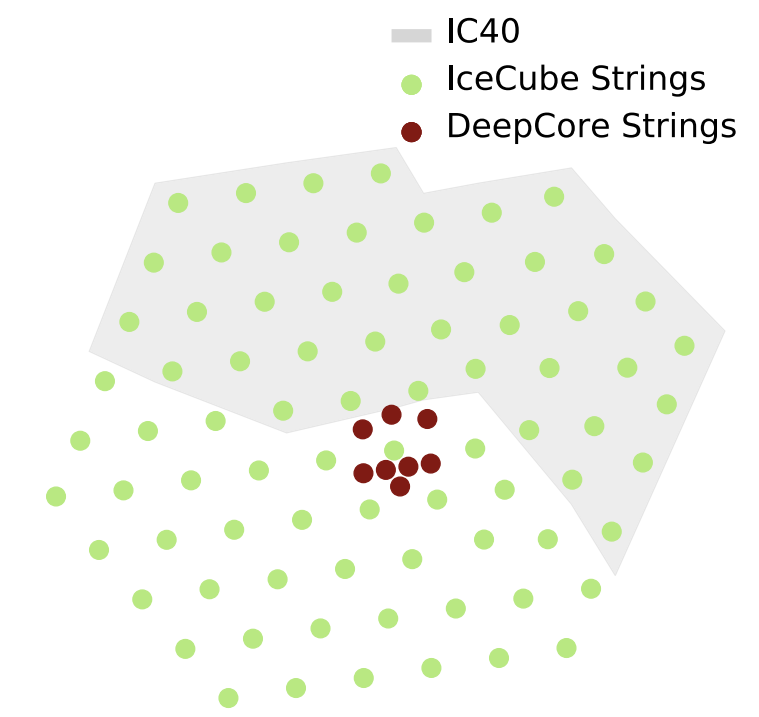

Fig. 1 A top view of the IceCube array. The IC40 configuration consists of all strings in the upper gray shaded area. After completion in the end of 2010, IceCube consists of all 86 strings, called the IC 86 configuration. DeepCore strings were excluded in the presented analyses

\section{IceCube}

The IceCube Neutrino Observatory is located at the geographic South Pole and consists of an in-ice array, IceCube [10], and a surface air shower array, IceTop [11], dedicated to neutrino and cosmic ray research, respectively. An aerial sketch of the detector layout is shown in Fig. 1.

IceCube consists of 86 strings with 60 digital optical modules (DOMs) each, deployed at depths between 1450 and $2450 \mathrm{~m}$, instrumenting a total volume of one cubic kilometer. Each DOM contains a $25 \mathrm{~cm}$ Hamamatsu photomultiplier tube (PMT) and electronics to read out and digitize the analog signal from the PMT [12]. The strings form a hexagonal grid with typical inter-string separation of $125 \mathrm{~m}$ and vertical DOM separation of $17 \mathrm{~m}$, except for six strings in the middle of the array that are more densely instrumented (with higher efficiency PMTs) and deployed closer together. These strings constitute the inner detector, DeepCore [13]. Construction of the IceCube detector started in December 2004 and was finished in December 2010, but the detector took data during construction. Specifically in this paper, we present results from two analyses, one performed with one year of data taken during 2008/2009, when the detector consisted of 40 strings, called IC40, and another analysis with data taken during 2011/2012 using the complete detector, called IC86.

IceCube uses natural ice both as target and as radiator. The analysis in the IC40 configuration of highly relativistic monopoles uses a six-parameter ice model [14] which describes the depth-dependent extrapolation of measurements of scattering and absorption valid for a wavelength 
of $400 \mathrm{~nm}$. The IC86 analysis of mildly relativistic monopoles uses an improved ice model which is based on additional measurements and accounts for different wavelengths [15].

Each DOM transmitted digitized PMT waveforms to the surface. The number of photons and their arrival times were then extracted from these waveforms. The detector is triggered when a DOM and its next or next-to-nearest DOMs record a hit within a $1 \mu$ s window. Then all hits in the detector within a window of $10 \mu \mathrm{s}$ will be read-out and combined into one event [16]. A series of data filters are run on-site in order to select potentially interesting events for further analysis, reducing at the same time the amount of data to be transferred via satellite. For both analyses presented here, a filter selecting events with a high number of photo-electrons ( $>650$ in the highly relativistic analysis and $>1000$ in the mildly relativistic analysis) were used. In addition filters selecting up-going track like events are used in the mildly relativistic analysis.

After the events have been sent to the IceCube's computer farm, they undergo some standard processing, such as the removal of hits which are likely caused by noise and basic reconstruction of single particle tracks via the LineFit algorithm [17]. This reconstruction is based on a 4-dimensional (position plus time) least-square fit which yields an estimated direction and velocity for an event.

The analyses are performed in a blind way by optimizing the cuts to select a possible monopole signal on simulation and one tenth of the data sample (the burn sample). The remaining data is kept untouched until the analysis procedure is fixed [18]. In the highly relativistic analysis the burn sample consists of all events recorded in August of 2008. In the mildly relativistic analysis the burn sample consists of every 10th 8-h-run in 2011/2012.

\section{Monopole signatures}

Magnetic monopoles can gain kinetic energy through acceleration in magnetic fields. This acceleration follows from a generalized Lorentz force law [20] and is analogous to the acceleration of electric charges in electric fields. The kinetic energy gained by a monopole of charge $g_{D}$ traversing a magnetic field $B$ with coherence length $L$ is $E \sim g_{D} B L$ [7]. This gives a gain of up to $10^{14} \mathrm{GeV}$ of kinetic energy in intergalactic magnetic fields to reach relativistic velocities. At such high kinetic energies magnetic monopoles can pass through the Earth while still having relativistic velocities when reaching the IceCube detector.

In the monopole velocity range considered in these analyses, $v \geq 0.4 c$ at the detector, three processes generate detectable light: direct Cherenkov emission by the monopole itself, indirect Cherenkov emission from ejected $\delta$-electrons and luminescence. Stochastical energy losses, such as pair production and photonuclear reactions, are neglected because they just occur at ultra-relativistic velocities.

An electric charge $e$ induces the production of Cherenkov light when its velocity $v$ exceeds the Cherenkov threshold $v_{C}=c / n_{P} \approx 0.76 c$ where $n_{P}$ is the refraction index of ice. A magnetic charge $g$ moving with a velocity $\beta=v / c$ produces an electrical field whose strength is proportional to the particle's velocity and charge. At velocities above $v_{C}$, Cherenkov light is produced analogous to the production by electrical charges [21] in an angle $\theta$ of

$$
\cos \theta=\frac{1}{n_{P} \beta}
$$

The number of Cherenkov photons per unit path length $x$ and wavelength $\lambda$ emitted by a monopole with one magnetic charge $g=g_{D}$ can be described by the usual Frank-Tamm formula [21] for a particle with effective charge $Z e \rightarrow g_{D} n_{P}$ [22]

$\frac{d^{2} N_{\gamma}}{d x d \lambda}=\frac{2 \pi \alpha}{\lambda^{2}}\left(\frac{g_{D} n_{P}}{e}\right)^{2}\left(1-\frac{1}{\beta^{2} n_{P}^{2}}\right)$

Thus, a minimally charged monopole generates $\left(g_{D} n_{P} / e\right)^{2}$ $\approx 8200$ times more Cherenkov radiation in ice compared to an electrically charged particle with the same velocity. This is shown in Fig. 2.

In addition to this effect, a (mildly) relativistic monopole knocks electrons off their binding with an atom. These highenergy $\delta$-electrons can have velocities above the Cherenkov threshold. For the production of $\delta$-electrons the differential cross-section of Kasama, Yang and Goldhaber (KYG) is used that allows to calculate the energy transfer of the monopole to the $\delta$-electrons and therefore the resulting output of indirect Cherenkov light $[23,24]$. The KYG cross section was calculated using QED, particularly dealing with the monopole's vector potential and its singularity [23]. Cross sections derived prior to KYG, such as the so-called Mott cross section [25-27], are only semi-classical approximations because the mathematical tools had not been developed by then. Thus, in this work the state-of-the-art KYG cross section is used to derive the light yield. The number of photons derived with the KYG and Mott cross section are shown in Fig. 2. Above the Cherenkov threshold indirect Cherenkov light is negligible for the total light yield.

Using the KYG cross section the energy loss of magnetic monopoles per unit path length $d E / d x$ can be calculated [28] 


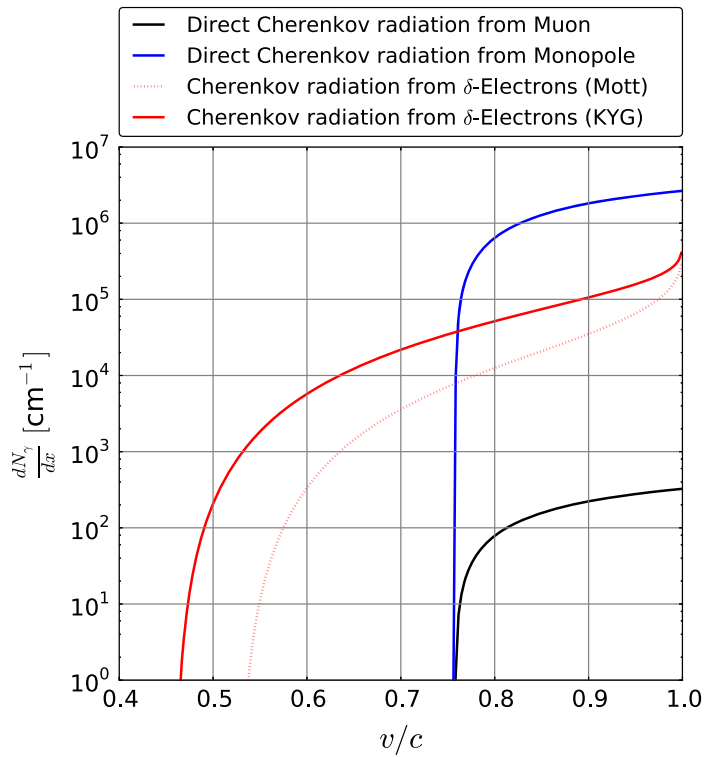

Fig. 2 Number of photons per $\mathrm{cm}$ produced by a muon (black), a monopole by direct Cherenkov light (blue), and monopoles by $\delta$ electrons. The photon yield per indirect Cherenkov light is shown using the KYG (red solid) and, for comparison, the Mott (red dotted) cross section, used in one earlier monopole analysis [19]. Light of wavelengths from 300 to $600 \mathrm{~nm}$ is considered here, covering the DOM acceptance of IceCube [15]

$$
\begin{aligned}
\frac{d E}{d x}= & \frac{4 \pi N_{e} g_{D}^{2} e^{2}}{m_{e} c^{2}}\left[\ln \frac{2 m_{e} c^{2} \beta^{2} \gamma^{2}}{I}+\frac{K\left(g_{D}\right)}{2}\right. \\
& \left.-\frac{\delta+1}{2}-B\left(g_{D}\right)\right]
\end{aligned}
$$

where $N_{e}$ is the electron density, $m_{e}$ is the electron mass, $\gamma$ is the Lorentz factor of the monopole, $I$ is the mean ionization potential, $K\left(g_{D}\right)$ is the QED correction derived from the KYG cross section, $B\left(g_{D}\right)$ is the Bloch correction and $\delta$ is the density-effect correction [29].

Luminescence is the third process which may be considered in the velocity range. It has been shown that pure ice exposed to ionizing radiation emits luminescence light $[30,31]$. The measured time distribution of luminescence light is fit well by several overlapping decay times which hints at several different excitation and de-excitation mechanisms [32]. The most prominent wavelength peaks are within the DOM acceptance of about 300-600 nm [15,32]. The mechanisms are highly dependent on temperature and ice structure. Extrapolating the latest measurements of luminescence light $d N_{\gamma} / d E$ [32,33], the brightness $d N_{\gamma} / d x$

$\frac{d N_{\gamma}}{d x}=\frac{d N_{\gamma}}{d E} \cdot \frac{d E}{d x}$

could be at the edge of IceCube's sensitivity where the energy loss is calculated with Eq. 4. This means that it would not be dominant above $0.5 c$. The resulting brightness is almost constant for a wide velocity range from 0.1 to $0.95 \mathrm{c}$. Depending on the actual brightness, luminescence light could be a promising method to detect monopoles with lower velocities. Since measurements of $d N_{\gamma} / d E$ are still to be done for the parameters given in IceCube, luminescence has to be neglected in the presented analyses which is a conservative approach leading to lower limits.

\section{Simulation}

The simulation of an IceCube event comprises several steps. First, a particle is generated, i.e. given its start position, direction and velocity. Then it is propagated, taking into account decay and interaction probabilities, and propagating all secondary particles as well. When the particle is close to the detector, the Cherenkov light is generated and the photons are propagated through the ice accounting for its properties. Finally the response of the PMT and DOM electronics is simulated including the generation of noise and the triggering and filtering of an event (see Sect. 2). From the photon propagation onwards, the simulation is handled identically for background and monopole signal. However the photon propagation is treated differently in the two analyses presented below due to improved ice description and photon propagation software available for the latter analysis.

\subsection{Background generation and propagation}

The background of a monopole search consists of all other known particles which are detectable by IceCube. The most abundant background are muons or muon bundles produced in air showers caused by cosmic rays. These were modeled using the cosmic ray models Polygonato [34] for the highly relativistic and GaisserH3a [35] for the mildly relativistic analysis.

The majority of neutrino induced events are caused by neutrinos created in the atmosphere. Conventional atmospheric neutrinos, produced by the decay of charged pions and kaons, are dominating the neutrino rate from the $\mathrm{GeV}$ to the $\mathrm{TeV}$ range [36]. Prompt neutrinos, which originate from the decay of heavier mesons, i.e. containing a charm quark, are strongly suppressed at these energies [37].

Astrophysical neutrinos, which are the primary objective of IceCube, have only recently been found $[38,39]$. For this reason they are only taken into account as a background in the mildly relativistic analysis, using the fit result for the astrophysical flux from Ref. [39].

Coincidences of all background signatures are also taken into account. 


\subsection{Signal generation and propagation}

Since the theoretical mass range for magnetic monopoles is broad (see Sect. 1), and the Cherenkov emission is independent of the mass, signal simulation is focused simply on a benchmark monopole mass of $10^{11} \mathrm{GeV}$ without limiting generality. Just the ability to reach the detector after passing through the Earth depends on the mass predicted by a monopole model. The parameter range for monopoles producing a recordable light emission inside IceCube is governed by the velocities needed to produce (indirect) Cherenkov light.

The starting points of the simulated monopole tracks are generated uniformly distributed around the center of the completed detector and pointing towards the detector. For the highly relativistic analysis the simulation could be run at specific monopole velocities only and so the characteristic velocities $0.76,0.8,0.9$ and $0.995 c$, were chosen.

Due to new software, described in the next sub-section, in the simulation for the mildly relativistic analysis the monopoles can be given an arbitrary characteristic velocity $v$ below $0.99 c$. The light yield from indirect Cherenkov light fades out below $0.5 c$. To account for the smallest detectable velocities the lower velocity limit was set to $0.4 c$ in simulation.

The simulation also accounts for monopole deceleration via energy loss. This information is needed to simulate the light output.

\subsection{Light propagation}

In the highly relativistic analysis the photons from direct Cherenkov light are propagated using Photonics [40]. A more recent and GPU-enabled software propagating light in IceCube is PPC [15] which is used in the mildly relativistic analysis. The generation of direct Cherenkov light, following Eq. 3, was implemented into PPC in addition to the variable Cherenkov cone angle (Eq. 2). For indirect Cherenkov light a parametrization of the distribution in Fig. 2 is used.

Both simulation procedures are consistent with each other and deliver a signal with the following topology: throughgoing tracks, originating from all directions, with constant velocities and brightness inside the detector volume, see Fig. 3. All these properties are used to discriminate the monopole signal from the background in IceCube.

\section{Highly relativistic analysis}

This analysis covers the velocities above the Cherenkov threshold $v_{C} \approx 0.76 c$ and it is based on the IC40 data recorded from May 2008 to May 2009. This comprises about 346 days of live-time or 316 days without the burn sample. The live-time is the recording time for clean data. The analysis for the IC40 data follows the same conceptual design as a

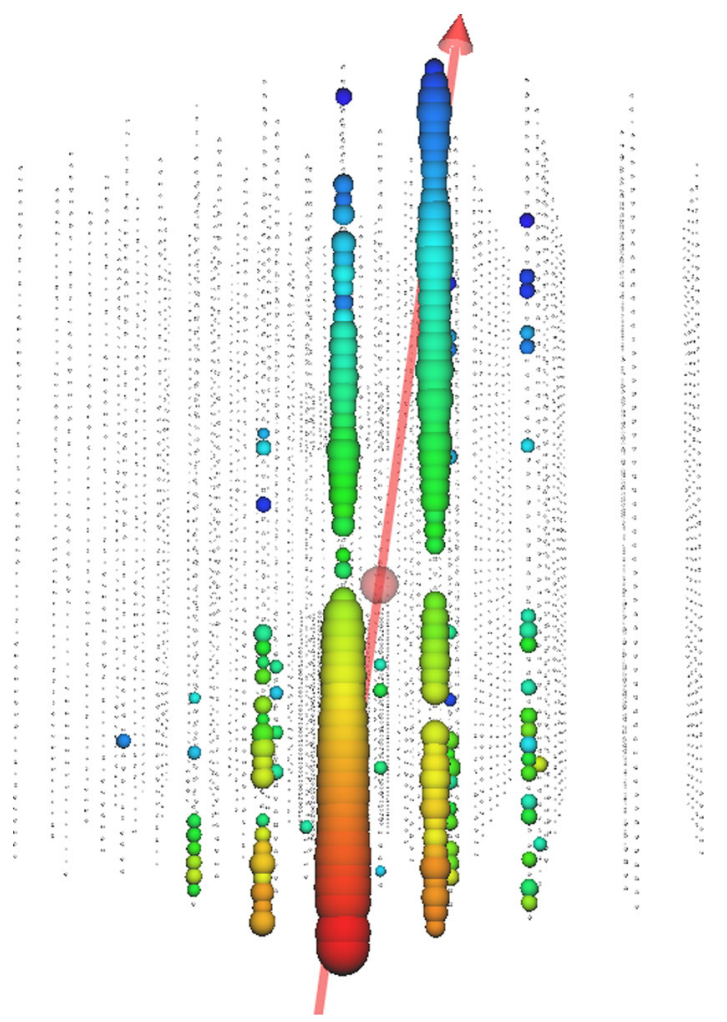

Fig. 3 Event view of a simulated magnetic monopole with a velocity of $0.83 c$ using both direct and indirect Cherenkov light. The monopole track is created with a zenith angle of about $170^{\circ}$ in upward direction. The position of the IceCube DOMs are shown with gray spheres. Hit DOMs are visualized with colored spheres. Their size is scaled with the number of recorded photons. The color denotes the time development from red to blue. The red line shows the reconstructed track which agrees with the true direction

previous analysis developed for the IC22 data [41], focusing on a simple and easy to interpret set of variables.

\subsection{Reconstruction}

The highly relativistic analysis uses spatial and timing information from the following sources: all DOMs, fulfilling the next or next-to-nearest neighbor condition (described in Sect. 2), and DOMs that fall into the topmost $10 \%$ of the collected-charge distribution for that event which are supposed to record less scattered photons. This selection allows definition of variables that benefit from either large statistics or precise timing information.

\subsection{Event selection}

The IC40 analysis selects events based on their relative brightness, arrival direction, and velocity. Some additional variables are used to identify and reject events with poor track reconstruction quality. The relative brightness is defined as the average number of photo-electrons per DOM contribut- 


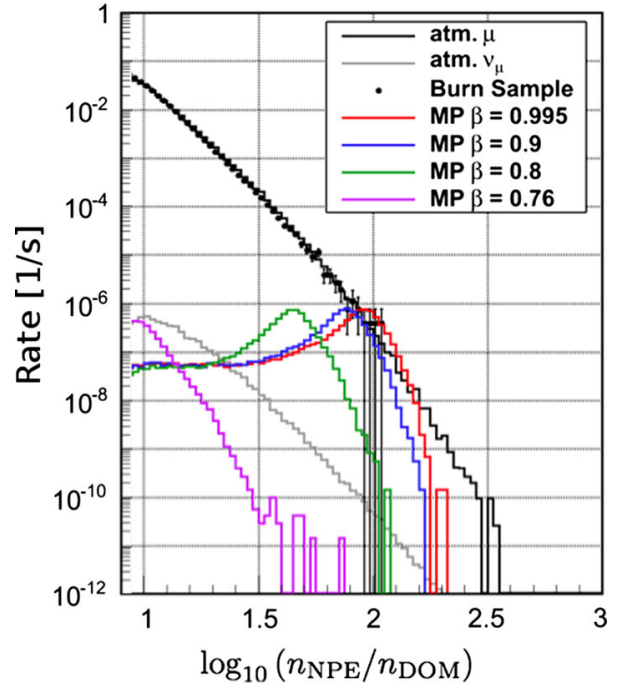

Fig. 4 The relative brightness after the first two cuts on $n_{\mathrm{DOM}}$ and $n_{\mathrm{NPE}} / n_{\mathrm{DOM}}$. The expected distributions from monopoles (MP) of different velocities is shown for comparison

ing to the event. This variable has more dynamic range compared with the number of hit DOMs. The distribution of this variable after applying the first two quality cuts, described in Table 3, is shown in Fig. 4. Each event selection step up to the final level is optimized to minimize the background passing rate while keeping high signal efficiency, see Table 3.

The final event selection level aims to remove the bulk of the remaining background, mostly consisting of downward going atmospheric muon bundles. However, the dataset is first split in two mutually exclusive subsets with low and high brightness. This is done in order to isolate a well known discrepancy between experimental and simulated data in the direction distribution near the horizon which is caused by deficiencies in simulating air shower muons at high inclinations [42].

Since attenuation is stronger at large zenith angles $\theta_{z}$, the brightness of the resulting events is reduced and the discrepancy is dominantly located in the low brightness subset. Only simulated monopoles with $v=0.76 c$ significantly populate this subset. The final selection criterion for the low brightness subset is $\cos \theta_{z}<-0.2$ where $\theta_{z}$ is the reconstructed arrival angle with respect to the zenith. For the high brightness subset a 2-dimensional selection criterion is used as shown in Fig. 5. The two variables are the relative brightness described above and the cosine of the arrival angle. Above the horizon $\left(\cos \theta_{z}>0\right)$, where most of the background is located, the selection threshold increases linearly with increasing $\cos \theta_{z}$. Below the horizon the selection has no directional dependence and values of both ranges coincide at $\cos \theta_{z}=0$. The optimization method applied here is the model rejection potential (MRP) method described in [41].

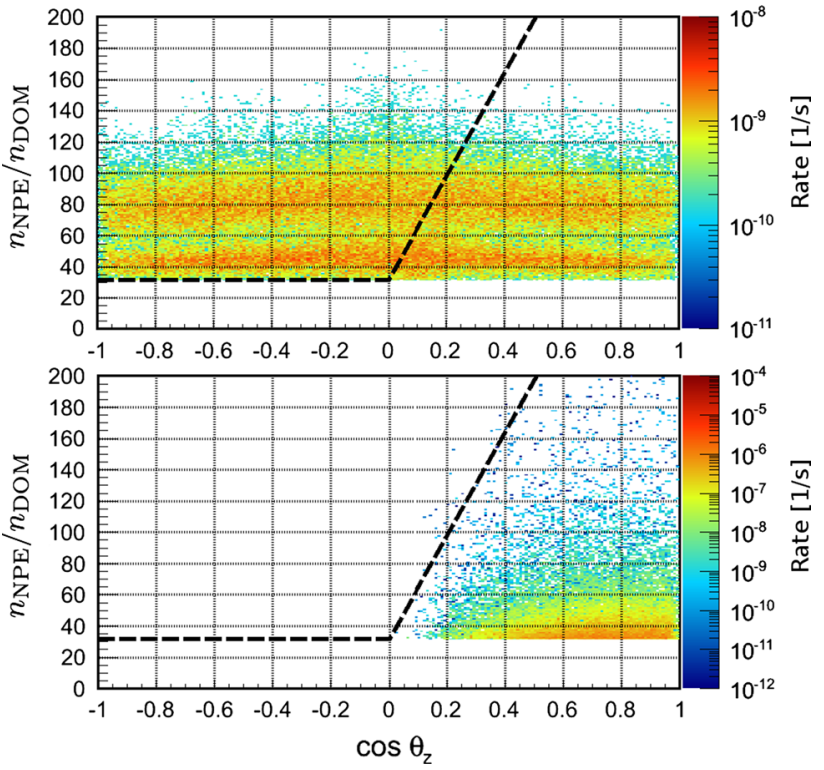

Fig. 5 Comparison of signal distribution (top) vs. atmospheric muon background (bottom) for the final cut. The signal is the composed out of the sum of monopoles with $\beta=0.995,0.9,0.8$

\subsection{Uncertainties and flux calculation}

Analogous to the optimization of the final event selection level, limits on the monopole flux are calculated using a MRP method. Due to the blind approach of the analysis these are derived from Monte Carlo simulations, which contain three types of uncertainties: (1) Theoretical uncertainties in the simulated models, (2) Uncertainties in the detector response, and (3) Statistical uncertainties.

For a given monopole-velocity the limit then follows from

$\Phi_{\alpha}=\operatorname{MRP} \cdot \Phi_{0}=\frac{\bar{\mu}_{\alpha}\left(n_{\mathrm{obs}}\right)}{\bar{n}_{\mathrm{s}}} \Phi_{0}$

where $\bar{\mu}_{\alpha}$ is an average Feldman-Cousins (FC) upper limit with confidence $\alpha$, which depends on the number of observed events $n_{\text {obs }}$. Similarly, though derived from simulation, $\bar{n}_{\mathrm{s}}$ is the average expected number of observed signal events assuming a flux $\Phi_{0}$ of magnetic monopoles. Since $\bar{n}_{\mathrm{S}}$ is proportional to $\Phi_{0}$ the final result is independent of whichever initial flux is chosen.

The averages can be independently expressed as weighted sums over values of $\mu_{\alpha}\left(n_{\mathrm{obs}}, n_{\mathrm{bg}}\right)$ and $n_{\mathrm{S}}$ respectively with the FC upper limit here also depending on the number of expected background events $n_{\mathrm{bg}}$ obtained from simulation. The weights are then the probabilities for observing a particular value for $n_{\mathrm{bg}}$ or $n_{\mathrm{s}}$. In the absence of uncertainties this probability has a Poisson distribution with the mean set to the expected number of events $\lambda$ derived from simulations. However, in order to extend the FC approach to account for uncertainties, the distribution 
$\operatorname{PDF}(n \mid \lambda, \sigma)=\int \frac{(\lambda+x)^{n} e^{-\lambda-x}}{n !} \cdot w(x \mid \sigma) d x$

is used instead to derive $n_{\mathrm{bg}}$ and $n_{\mathrm{s}}$. This is the weighted average of Poisson distributions where the mean value varies around the central value $\lambda$ and the variance $\sigma^{2}$ is the quadratic sum of all individual uncertainties. Under the assumption that individual contributions to the uncertainty are symmetric and independent, the weighting function $w(x \mid \sigma)$ is a normal distribution with mean 0 and variance $\sigma^{2}$. However, the Poisson distribution is only defined for positive mean values. Therefore a truncated normal distribution with the boundaries $-\lambda$ and $+\infty$ is used as the weighting function instead.

\section{Mildly relativistic analysis}

This analysis uses the data recorded from May 2011 to May 2012. It comprises about 342 days ( 311 days without the burn sample) of live-time. The signal simulation covers the velocity range of $0.4-0.99 c$. The optimization of cuts and machine learning is done on a limited velocity range $<0.76 c$ to focus on lower velocities where indirect Cherenkov light dominates.

\subsection{Reconstruction}

Following the filters, described in Sect. 2, further processing of the events is done by splitting coincident events into subevents using a time-clustering algorithm. This is useful to reject hits caused by PMT after-pulses which appear several microseconds later than signal hits.

For quality reasons events are required to have 6 DOMs on 2 strings hit, see Table 4 . The remaining events are handled as tracks reconstructed with an improved version [17] of the LineFit algorithm, mentioned in Sect. 2. Since the main background in IceCube are muons from air showers which cause a down-going track signature, a cut on the reconstructed zenith angle below $86^{\circ}$ removes most of this background.

Figure 6 shows the reconstructed particle velocity at this level. The rate for atmospheric muon events has its maximum at low velocities. This is due to mostly coincident events remaining in this sample. The muon neutrino event rate consists mainly of track-like signatures and is peaked at the velocity of light. Dim events or events traversing only part of the detector are reconstructed with lower velocities which leads to the smearing of the peak rate for muon neutrinos and monopole simulations. Electron neutrinos usually produce a cascade of particles (and light) when interacting which is easy to separate from a track signature. The velocity reconstruction for these events results mainly in low velocities which can also be used for separation from signal.
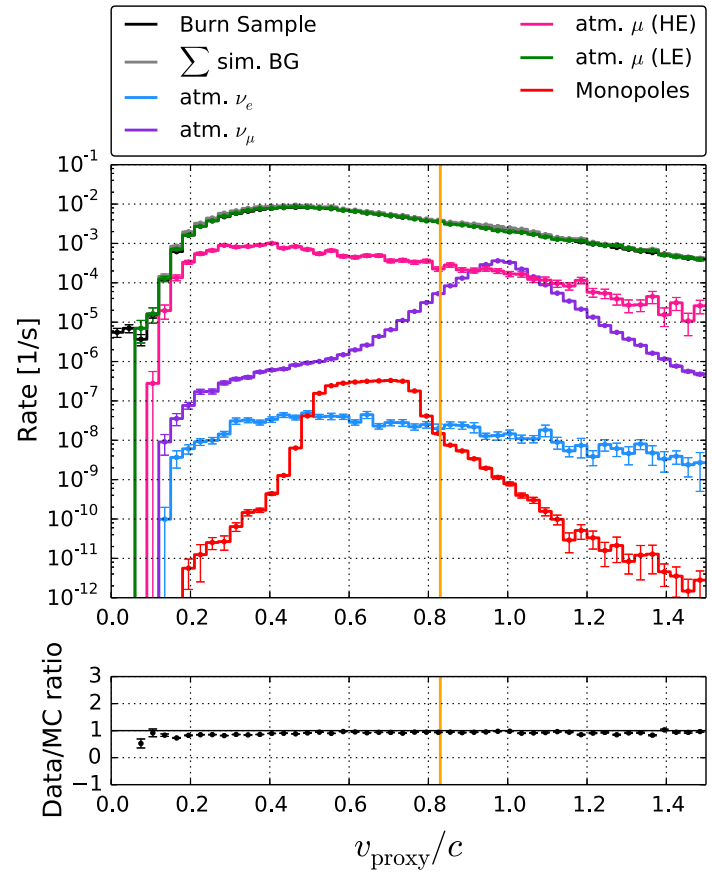

Fig. 6 Estimated velocity after event reconstruction. In this plot only monopoles with a simulated true velocity below $0.76 c$ are shown and a cut on the reconstructed velocity at $0.83 c$. These restrictions were only used for training to focus on this range and released for sensitivity calculation and unblinding. Superluminal velocity values occur because of the simplicity of the chosen reconstruction algorithm which may lead to mis-reconstructed events that can be discarded. The air shower background is divided into high $(H E)$ and low energy $(L E)$ primary particle energy at $100 \mathrm{TeV}$. The recorded signals differ significantly and are therefore treated with different variables and cuts

\subsection{Event selection}

In contrast to the highly relativistic analysis, machine learning was used. A boosted decision tree (BDT) [43] was chosen to account for limited background statistics. The multivariate method was embedded in a re-sampling method. This was combined with additional cuts to reduce the background rate and prepare the samples for an optimal training result. Besides that, these straight cuts reduce cascades, coincident events, events consisting of pure noise, improve reconstruction quality, and remove short tracks which hit the detector at the edges. See a list of all cuts in Table 4. To train the BDT on lower velocities an additional cut on the maximal velocity $0.82 c$ is used only during training which is shown in Fig. 6 . Finally a cut on the penetration depth of a track, measured from the bottom of the detector, is performed. This is done to lead the BDT training to a suppression of air shower events underneath the neutrino rate near the signal region, as can be seen in Fig. 8.

Out of a the large number of variables provided by standard and monopole reconstructions 15 variables were chosen for the BDT using a tool called mRMR (Minimum Redundancy Maximum Relevance) [44]. These 15 variables are 


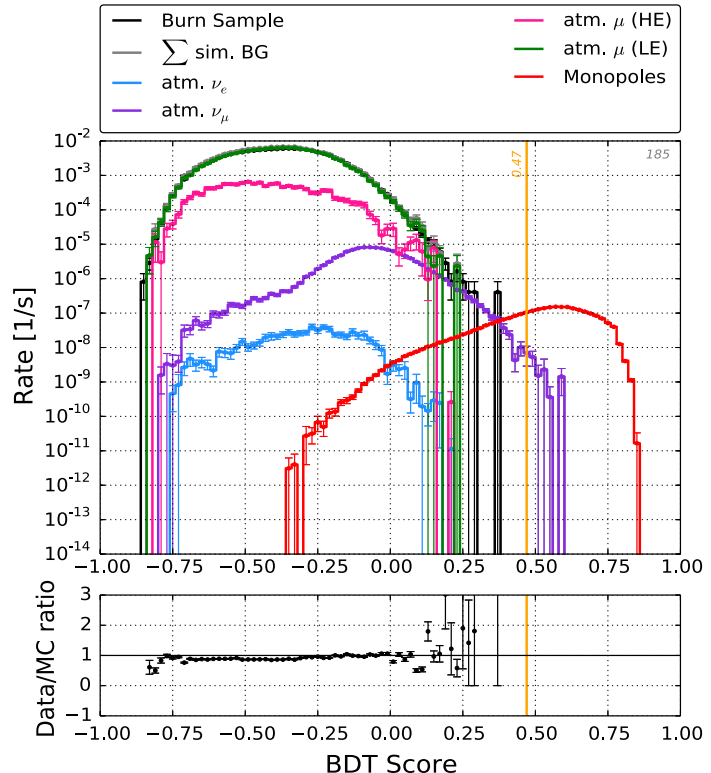

Fig. 7 Distribution of one BDT trained on $10 \%$ of the burn sample. The cut value which is chosen using Fig. 8 is shown with the orange line. Statistical errors per bin are drawn

described in Table 5. With regard to the next step it was important to choose variables which show a good data - simulation agreement so that the BDT would not be trained on unknown differences between simulation and recorded data. The resulting BDT score distribution in Fig. 7 shows a good signal vs. background separation with reasonable simulation - data agreement. The rate of atmospheric muons and electron neutrinos induced events is suppressed sufficiently compared to the muon neutrino rate near the signal region. The main background is muon neutrinos from air showers.

\subsection{Background expectation}

To calculate the background expectation a method inspired by bootstrapping is used [45], called pull-validation [46]. Bootstrapping is usually used to smooth a distribution by resampling the limited available statistics. Here, the goal is to smooth especially the tail near the signal region in Fig. 7.

Usually $50 \%$ of the available data is chosen to train a BDT which is done here just for the signal simulation. Then the other $50 \%$ is used for testing. Here, $10 \%$ of the burn sample are chosen randomly, to be able to consider the variability in the tails of the background.

Testing the BDT on the other $90 \%$ of the burn sample leads to an extrapolation of the tail into the signal region. This re-sampling and BDT training/testing is repeated 200 times, each time choosing a random $10 \%$ sample. In Fig. 8 the bin-wise average and standard deviation of 200 BDT score distributions are shown.

By BDT testing, 200 different BDT scores are assigned to each single event. The event is then transformed into a prob-

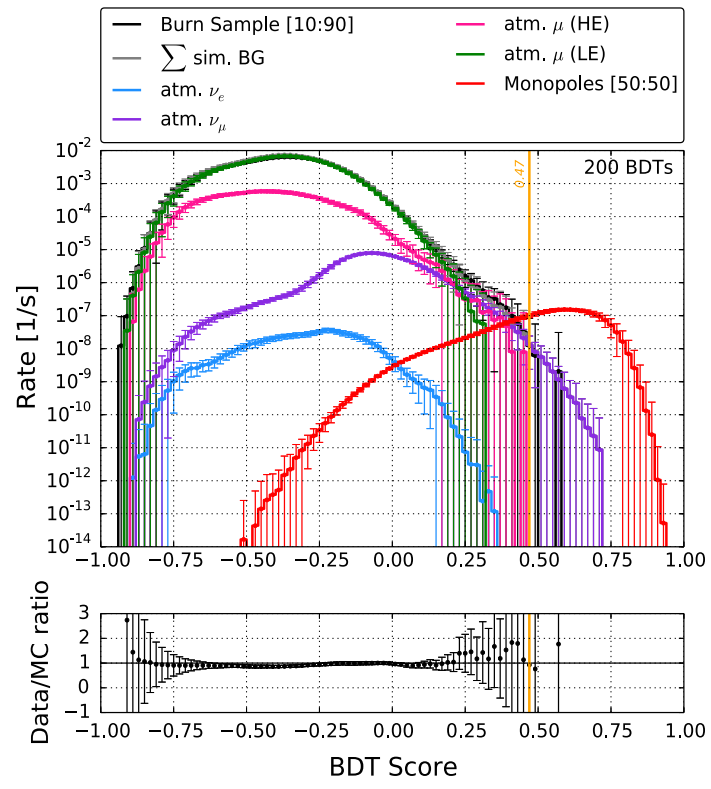

Fig. 8 Average of 200 BDTs. An example of one contributing BDT is shown in Fig. 7. In each bin the mean bin height in 200 BDTs is shown with the standard deviation as error bar. Based on this distribution the MRF is calculated and minimized to choose the cut value

ability density distribution. When cutting on the BDT score distribution in Fig. 8 a single event $i$ is neither completely discarded nor kept, but it is kept with a certain probability $p_{i}$ which is calculated as a weight. The event is then weighted in total with $W_{i}=p_{i} \cdot w_{i}$ using its survival probability and the weight $w_{i}$ from the chosen flux spectrum. Therefore, many more events contribute to the cut region compared to a single BDT which reduces the uncertainty of the background expectation.

To keep the error of this statistical method low, the cut on the averaged BDT score distribution is chosen near the value where statistics in a single BDT score distribution vanishes.

The developed re-sampling method gives the expected background rate including an uncertainty for each of the single BDTs. Therefore one BDT was chosen randomly for the unblinding of the data.

\subsection{Uncertainties}

The uncertainties of the re-sampling method were investigated thoroughly. The Poissonian error per bin is negligible because of the averaging of 200 BDTs. Instead, there are 370 partially remaining events which contribute to the statistical error. This uncertainty $\Delta_{\text {contr }}$ is estimated by considering the effect of omitting individual events $i$ of the 370 events from statistics

$\Delta_{\text {contr }}=\max _{i}\left(\frac{w_{i} p_{i}}{\sum_{i} w_{i} p_{i}}\right)$ 
Fig. 9 Sensitivities (magenta) and final limits (red) of both analysis at certain characteristic velocities compared to other limits. The lines are only drawn to guide the eyes. Other limits are from BAIKAL [33], ANTARES [19], IceCube 22 [41], MACRO [48]. Also shown is the Parker limit described in the text [49]

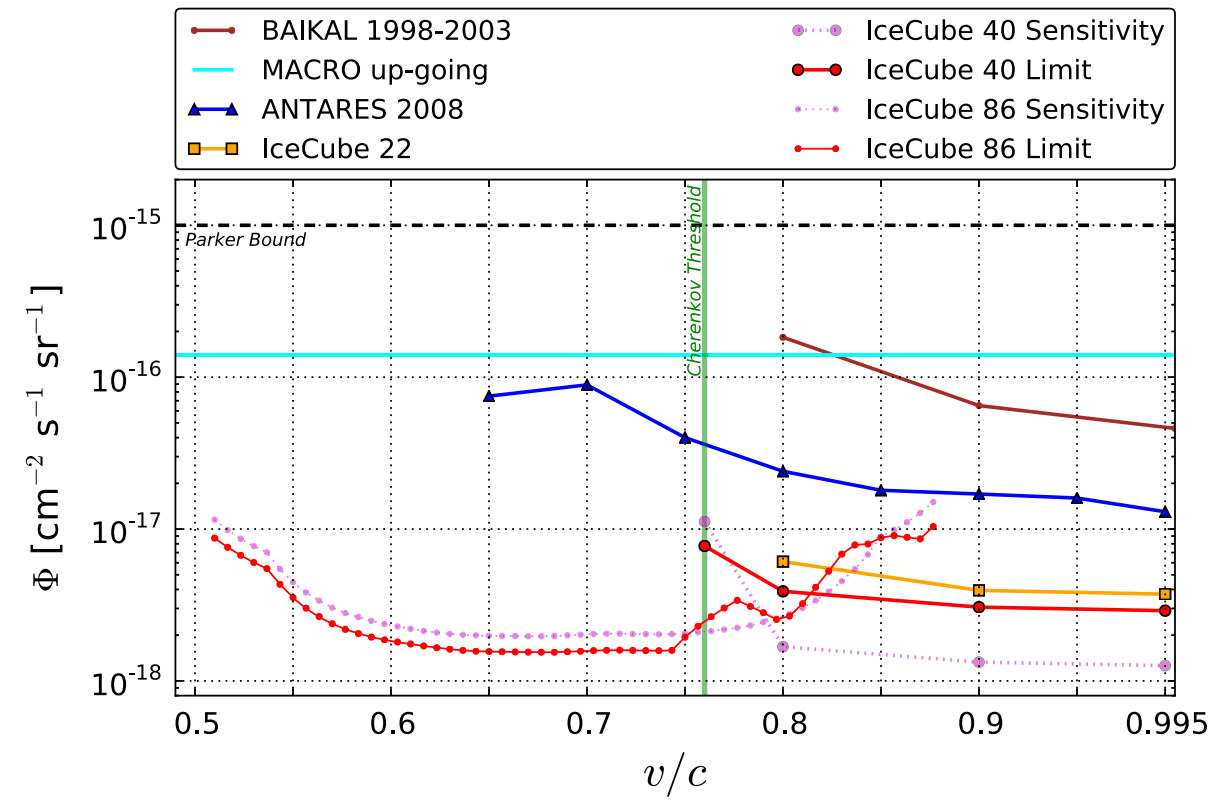

Datasets with different simulation parameters for the detector properties are used to calculate the according uncertainties. The values of all calculated uncertainties are shown in Table 1.

The robustness of the re-sampling method was verified additionally by varying all parameters and cut values of the analysis. Several fake unblindings were done by training the analysis on a $10 \%$ sample of the burn sample, optimizing the last cut and then applying this event selection on the other $90 \%$ of the burn sample. This proves reliability by showing that the previously calculated background expectation is actually received with increase of statistics by one order of magnitude. The results were mostly near the mean neutrino rate, only few attempts gave a higher rate, but no attempt exceeded the calculated confidence interval.

The rate of the background events has a variability in all 200 BDTs of up to 5 times the mean value of 0.55 events per live-time (311 days) when applying the final cut on the BDT score. This contribution is dominating the total uncertainties. Therefore not a normal distribution but the real distribution is used for further calculations. This distribution is used as a probability mass function in an extended Feldman Cousin approach to calculate the $90 \%$ confidence interval, as described in Sect. 5.3. The final cut at BDT score 0.47 is chosen near the minimum of the model rejection factor (MRF) [47]. To reduce the influence of uncertainties it was shifted to a slightly lower value. The sensitivity for many different velocities is calculated as described in Sect. 5.3 and shown in Fig. 9. This gives an $90 \%$ confidence upper limit of 3.61 background events. The improvement of sensitivity compared to recent limits by ANTARES [19] and MACRO [48] reaches from one to almost two orders of magnitude which reflects a huge detection potential.

\section{Results}

After optimizing the two analyses on the burn samples, the event selection was adhered to and the remaining $90 \%$ of the experimental data were processed ("unblinded"). The corresponding burn samples were not included while calculating the final limits.

\subsection{Result of the highly relativistic analysis}

In the analysis based on the IC40 detector configuration three events remain, one in the low brightness subset and two in the high brightness subset. The low brightness event is consistent with a background- only observation with 2.2 expected background events. The event itself shows characteristics typical for a neutrino induced muon. For the high brightness subset, with an expected background of 0.1 events, the observation of two events apparently contradicts the background-only hypothesis. However, a closer analysis of the two events reveals that they are unlikely to be caused by monopoles. These very bright events do not have a track like signature but a spheric development only partly contained in the detector. A possible explanation is the now established flux of cosmic neutrinos which was not included in the background expectation for this analysis. IceCube's unblinding policy prevents any claims on these events or reanalysis with changed cuts as have been employed with IC22 [41]. Instead they are treated as an upward fluctuation of the background weakening the limit. The final limits outperform previous limits and are shown in Table 2 and Fig. 9. These limits can also be used as a conservative limit for $v>0.995 c$ without optimization for high values of Lorentz factor $\gamma$ as the 


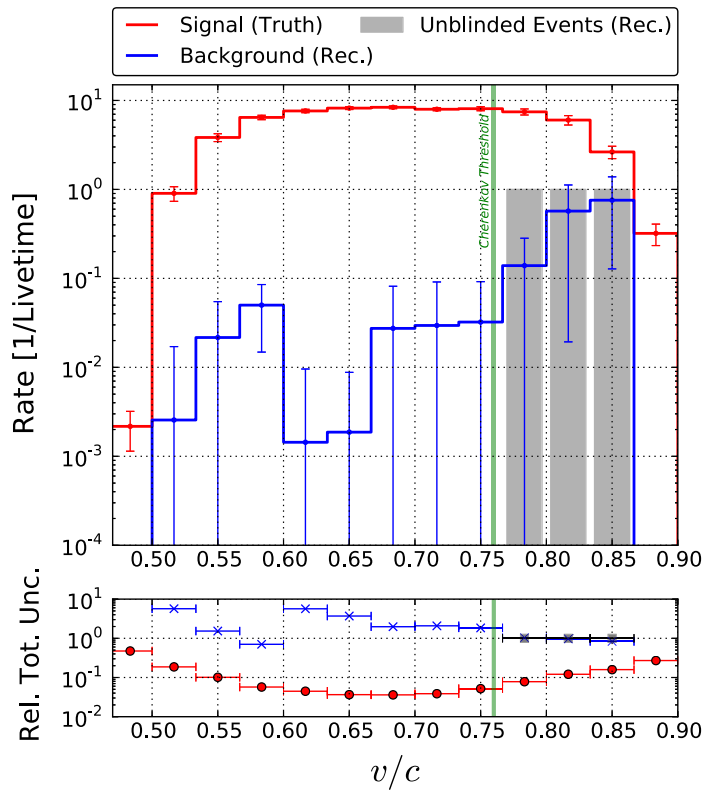

Fig. 10 Signal and background rates per characteristic monopole velocity which are used to calculate the final limits. Reconstructed velocity is used for background and true simulated velocity for signal. The lower part of the plot shows the velocity dependence of the uncertainties including the re-sampling uncertainty which dominates. The different contributions to the uncertainties are listed in Table 1

expected monopole signal is even brighter due to stochastic energy losses which are not considered here.

\subsection{Result of the mildly relativistic analysis}

In the mildly relativistic analysis three events remain after all cuts which is within the confidence interval of up to 3.6 events and therefore consistent with a background only observation. All events have reconstructed velocities above the training region of $0.76 c$. This is compared to the expectation from simulation in Fig. 10. Two of the events show a signature which is clearly incompatible with a monopole signature when investigated by eye because they are stopping within the detector volume. The third event, shown in Fig. 11 , may have a mis-reconstructed velocity due to the large string spacing of IceCube. However, its signature is comparable with a monopole signature with a reduced light yield than described in Sect. 3. According to simulations, a monopole of this reconstructed velocity would emit about 6 times the observed light.

To be comparable to the other limits shown in Fig. 9 the final result of this analysis is calculated for different characteristic monopole velocities at the detector. The bin width of the velocity distribution in Fig. 10 is chosen to reflect the error on the velocity reconstruction. Then, the limit in each bin is calculated and normalized which gives a step function. To avoid the bias on a histogram by choosing different histogram origins, five different starting points are chosen for

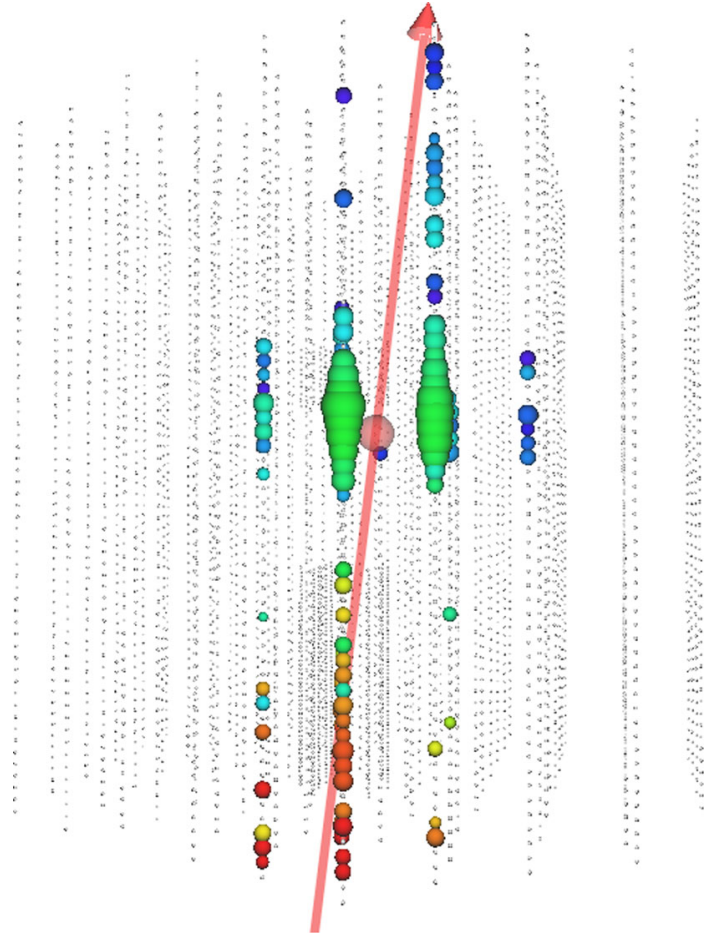

Fig. 11 One of the three events which were selected in the mildly relativistic analysis with a BDT Score of 0.53 . The reconstructed parameters of this event are the same as in Fig. 3. In this event, 110 DOMs were hit on 8 strings. It has a brightness of 595 NPE and causes an after-pulse. The position of the IceCube DOMs are shown with small gray spheres. Hit DOMs are visualized with colored spheres. Their size is scaled with the brightness of the hit. The color denotes the time development from red to blue. The red line shows the reconstructed track

the distribution in Fig. 10 and the final step functions are averaged [50].

The final limit is shown in Fig. 9 and Table 2 together with the limits from the highly relativistic analysis and other recent limits.

\section{Discussion}

The resulting limits are placed into context by considering indirect theoretical limits and previous experimental results. The flux $\Phi$ of magnetic monopoles can be constrained model independently by astrophysical arguments to $\Phi_{\mathrm{P}} \leq 10^{-15}$ $\mathrm{cm}^{-2} \mathrm{~s}^{-1} \mathrm{sr}^{-1}$ for a monopole mass below $10^{17} \mathrm{GeV} / c^{2}$. This value is the so-called Parker bound [49] which has already been surpassed by several experiments as shown in Fig. 9. The most comprehensive search for monopoles, regarding the velocity range, was done by the MACRO collaboration using different detection methods [48].

More stringent flux limits have been imposed by using larger detector volumes, provided by high-energy neutrino telescopes, such as ANTARES [19], BAIKAL [33], AMANDA [51], and IceCube [41]. The current best limits for non- 
relativistic velocities $(\leq 0.1 \mathrm{c})$ have been established by IceCube, constraining the flux down to a level of $\Phi_{90 \%} \geq$ $10^{-18} \mathrm{~cm}^{-2} \mathrm{~s}^{-1} \mathrm{sr}^{-1}$ [52]. These limits hold for the proposal that monopoles catalyze proton decay. The analysis by ANTARES is the only one covering the mildly relativistic velocity range ( $\geq 0.625 \mathrm{c}$ ) using a neutrino detector, to date. However, using the KYG cross section for the $\delta$-electron production would extend their limits to lower velocities. The Baksan collaboration has also produced limits on a monopole flux [53], both at slow and relativistic velocities, although due to its smaller size their results are not competitive with the results shown in Fig. 9.

\section{Summary and outlook}

We have described two searches using IceCube for cosmic magnetic monopoles for velocities $>0.51 \mathrm{c}$. One analysis focused on high monopole velocities at the detector $v>0.76 c$ where the monopole produces Cherenkov light and the resulting detector signal is extremely bright. The other analysis considers lower velocities $>0.51 \mathrm{c}$ where the monopole induces the emission of Cherenkov light in an indirect way and the brightness of the final signal is decreasing largely with lower velocity. Both analyses use geometrical information in addition to the velocity and brightness of signals to suppress background. The remaining events after all cuts were identified as background. Finally the analyses bound the monopole flux to nearly two orders of magnitude below previous limits. Further details of these analyses are given in Refs. [42,54].

Comparable sensitivities are expected from the future KM3NeT instrumentation based on scaling the latest ANTARES limit to a larger effective volume [55]. Also an ongoing ANTARES analysis plans to use six years of data and estimates competitive sensitivities for highly relativistic velocities [56].

Even better sensitivities are expected from further years of data taking with IceCube, or from proposed volume exten- sions of the detector [57]. A promising way to extend the search to slower monopoles with $v \leq 0.5 c$ is to investigate the luminescence they would generate in ice which may be detectable using the proposed low energy infill array PINGU [58].

Acknowledgments We acknowledge the support from the following agencies: U.S. National Science Foundation-Office of Polar Programs, U.S. National Science Foundation-Physics Division, University of Wisconsin Alumni Research Foundation, the Grid Laboratory Of Wisconsin (GLOW) grid infrastructure at the University of Wisconsin - Madison, the Open Science Grid (OSG) grid infrastructure; U.S. Department of Energy, and National Energy Research Scientific Computing Center, the Louisiana Optical Network Initiative (LONI) grid computing resources; Natural Sciences and Engineering Research Council of Canada, WestGrid and Compute/Calcul Canada; Swedish Research Council, Swedish Polar Research Secretariat, Swedish National Infrastructure for Computing (SNIC), and Knut and Alice Wallenberg Foundation, Sweden; German Ministry for Education and Research (BMBF), Deutsche Forschungsgemeinschaft (DFG), Helmholtz Alliance for Astroparticle Physics (HAP), Research Department of Plasmas with Complex Interactions (Bochum), Germany; Fund for Scientific Research (FNRSFWO), FWO Odysseus programme, Flanders Institute to encourage scientific and technological research in industry (IWT), Belgian Federal Science Policy Office (Belspo); University of Oxford, United Kingdom; Marsden Fund, New Zealand; Australian Research Council; Japan Society for Promotion of Science (JSPS); the Swiss National Science Foundation (SNSF), Switzerland; National Research Foundation of Korea (NRF); Danish National Research Foundation, Denmark (DNRF).

Open Access This article is distributed under the terms of the Creative Commons Attribution 4.0 International License (http://creativecomm ons.org/licenses/by/4.0/), which permits unrestricted use, distribution, and reproduction in any medium, provided you give appropriate credit to the original author(s) and the source, provide a link to the Creative Commons license, and indicate if changes were made. Funded by $\mathrm{SCOAP}^{3}$.

\section{Appendix}

In Table 1 the uncertainties of both analyses are shown. Table 2 gives the numeric values of the derived limits of both analyses. Tables 3, 4 and 5 show the event selection of both analyses in detail which illustrates how magnetic monopoles can be separated from background signals in IceCube.

Table 1 Uncertainties in both analyses. For the mildly relativistic analysis the average for the whole velocity range is shown. See Fig. 10 for the velocity dependence

\begin{tabular}{|c|c|c|c|c|c|c|c|c|}
\hline \multirow{3}{*}{$\begin{array}{l}\text { Conf. } \\
\text { Type }\end{array}$} & \multicolumn{6}{|l|}{$\mathrm{IC} 40$} & \multicolumn{2}{|l|}{ IC86 } \\
\hline & \multicolumn{2}{|l|}{ Atm. $v_{\mu}$ in $\%$} & \multicolumn{4}{|l|}{ Signal in $\%$} & $v_{\mu}$ in $\%$ & Signal in \% \\
\hline & High $n_{\mathrm{NPE}} / n_{\mathrm{DOM}}$ & Low $n_{\mathrm{NPE}} / n_{\mathrm{DOM}}$ & $\beta=0.995$ & $\beta=0.9$ & $\beta=0.8$ & $\beta=0.76$ & \multicolumn{2}{|c|}{$0.4 \leq \beta \leq 0.99$} \\
\hline Statistics & 3.7 & 6.4 & 0.7 & 0.7 & 0.8 & 0.5 & 6.8 & 0.4 \\
\hline DOM efficiency & 25.9 & 40.8 & 3.2 & 2.7 & 5.3 & 15.6 & 8.1 & 1.3 \\
\hline Light propagation & 20.5 & 34.9 & 2.9 & 2.4 & 3.6 & 6.1 & 12.4 & 2.7 \\
\hline Flux & 25.8 & 26.1 & - & - & - & - & 8.2 & - \\
\hline Re-sampling & - & - & - & - & - & - & See text & See text \\
\hline Total & 42.1 & 60.0 & 4.4 & 3.7 & 6.4 & 16.7 & 16.9 & 3.0 \\
\hline
\end{tabular}


Table 2 Values of final limits of both analyses

\begin{tabular}{|c|c|c|c|c|}
\hline Conf. & Velocity $[v / c]$ & $\begin{array}{l}\Phi_{90 \%} / 10^{-18} \\
{\left[\mathrm{~cm}^{-2} \mathrm{~s}^{-1} \mathrm{sr}^{-1}\right]}\end{array}$ & $\begin{array}{l}\text { Velocity }[v / c] \\
\text { (cont.) }\end{array}$ & $\begin{array}{l}\Phi_{90 \%} / 10^{-18} \\
{\left[\mathrm{~cm}^{-2} \mathrm{~s}^{-1} \mathrm{sr}^{-1}\right] \text { (cont.) }}\end{array}$ \\
\hline \multirow[t]{2}{*}{ IC40 } & 0.76 & 7.73 & 0.8 & 3.89 \\
\hline & 0.9 & 3.06 & 0.995 & 2.90 \\
\hline \multirow[t]{28}{*}{ IC86 } & 0.510 & 8.71 & 0.517 & 7.58 \\
\hline & 0.523 & 6.71 & 0.530 & 6.02 \\
\hline & 0.537 & 5.49 & 0.543 & 4.33 \\
\hline & 0.550 & 3.54 & 0.557 & 3.01 \\
\hline & 0.563 & 2.66 & 0.570 & 2.38 \\
\hline & 0.577 & 2.18 & 0.583 & 2.05 \\
\hline & 0.590 & 1.94 & 0.597 & 1.86 \\
\hline & 0.603 & 1.80 & 0.610 & 1.75 \\
\hline & 0.617 & 1.70 & 0.623 & 1.65 \\
\hline & 0.630 & 1.62 & 0.637 & 1.59 \\
\hline & 0.643 & 1.57 & 0.650 & 1.56 \\
\hline & 0.657 & 1.56 & 0.663 & 1.55 \\
\hline & 0.670 & 1.55 & 0.677 & 1.55 \\
\hline & 0.683 & 1.54 & 0.690 & 1.56 \\
\hline & 0.697 & 1.57 & 0.703 & 1.58 \\
\hline & 0.710 & 1.59 & 0.717 & 1.59 \\
\hline & 0.723 & 1.59 & 0.730 & 1.58 \\
\hline & 0.737 & 1.58 & 0.743 & 1.59 \\
\hline & 0.750 & 1.94 & 0.757 & 2.29 \\
\hline & 0.763 & 2.65 & 0.770 & 3.02 \\
\hline & 0.777 & 3.39 & 0.783 & 3.10 \\
\hline & 0.790 & 2.81 & 0.797 & 2.54 \\
\hline & 0.803 & 2.67 & 0.810 & 3.23 \\
\hline & 0.817 & 4.14 & 0.823 & 5.28 \\
\hline & 0.830 & 6.84 & 0.837 & 7.85 \\
\hline & 0.843 & 7.97 & 0.850 & 8.77 \\
\hline & 0.857 & 9.05 & 0.863 & 8.82 \\
\hline & 0.870 & 8.61 & 0.877 & 10.39 \\
\hline
\end{tabular}

Table 3 Description of all cuts in the highly relativistic analysis. For some cuts only the $10 \%$ of the DOMs with the highest charge (HC) were chosen

\begin{tabular}{|c|c|c|c|c|}
\hline Cut variable & Cut value & Hits & Description & Motivation \\
\hline$n_{\mathrm{DOM}}$ & $>60$ & All & Number of hit DOMs & $\begin{array}{l}\text { Improve quality of } n_{\mathrm{NPE}} / n_{\mathrm{DOM}} \\
\text { variable }\end{array}$ \\
\hline$n_{\mathrm{NPE}} / n_{\mathrm{DOM}}$ & $\geq 8$ & All & Average number of photo-electrons per DOM & $\begin{array}{l}\text { Reduce events with low relative } \\
\text { brightness }\end{array}$ \\
\hline$v$ & $\geq 0.72 \mathrm{c}$ & $\mathrm{HC}$ & Reconstructed velocity & Reduce cascade events \\
\hline$n_{\text {String }}$ & $\geq 2$ & $\mathrm{HC}$ & Number of hit strings & Reduce cascade events \\
\hline$t$ & $\geq 792 \mathrm{~ns}$ & $\mathrm{HC}$ & $\begin{array}{l}\text { Time length of an event; calculated by ordering } \\
\text { all hits in time and subtracting the last minus } \\
\text { the first time value }\end{array}$ & Reduce cascade events \\
\hline Topological & No split & All & $\begin{array}{l}\text { Attempt to sort the hits in an event into } \\
\text { topologically connected sets }\end{array}$ & Split coincident events \\
\hline Trigger $\mathrm{NHF}_{100}$ & $<0.784$ & All & $\begin{array}{l}\text { Fraction of DOMs with no hit in a } 100 \mathrm{~m} \\
\text { cylinder radius around the reconstructed track }\end{array}$ & $\begin{array}{l}\text { Reduce (coincident/noise) events } \\
\text { with spurious reconstruction }\end{array}$ \\
\hline
\end{tabular}


Table 3 continued

\begin{tabular}{|c|c|c|c|c|}
\hline Cut variable & Cut value & Hits & Description & Motivation \\
\hline$d_{\perp}$ & $<\left(110-64 \cdot \mathrm{NHF}_{100}\right) \mathrm{m}$ & All & $\begin{array}{l}\text { Root mean square of the lateral distance of hit } \\
\text { DOMs (weighted with DOM charge) from the } \\
\text { track }\end{array}$ & $\begin{array}{l}\text { Reduce (coincident/noise) } \\
\text { events with spurious } \\
\text { reconstruction }\end{array}$ \\
\hline$d_{\text {Gap } 100}$ & $\leq 420 \mathrm{~m}$ & All & $\begin{array}{l}\text { The maximal length of the track, which got no } \\
\text { hits within the specified track cylinder radius in } \\
\text { meters }\end{array}$ & $\begin{array}{l}\text { Reduce (coincident/noise) } \\
\text { events with spurious } \\
\text { reconstruction }\end{array}$ \\
\hline \multicolumn{5}{|c|}{ Low brightness cuts $\left(n_{\mathrm{NPE}} / n_{\mathrm{DOM}}<31.62\right)$} \\
\hline$t$ & $>1400 \mathrm{~ns}$ & $\mathrm{HC}$ & See above & See above (hardened cut) \\
\hline$d_{\text {Gap } 100}$ & $>112$ and $<261 \mathrm{~m}$ & All & See above & See above (hardened cut) \\
\hline $\cos \theta$ & $<-0.2$ & $\mathrm{HC}$ & Reconstructed zenith angle & $\begin{array}{l}\text { Reduce events caused by } \\
\text { mostly downward moving } \\
\text { air shower muons }\end{array}$ \\
\hline \multicolumn{5}{|c|}{ High brightness cuts $\left(n_{\mathrm{NPE}} / n_{\mathrm{DOM}} \geq 31.62\right)$} \\
\hline$t$ & $\geq(792+2500 \cdot \cos \theta) \mathrm{ns}$ & $\mathrm{HC}$ & See above & $\begin{array}{l}\text { Reduce events caused by } \\
\text { mostly downward moving } \\
\text { air shower muons } \\
\text { (supportive cut) }\end{array}$ \\
\hline$n_{\mathrm{NPE}} / n_{\mathrm{DOM}}$ & $\geq 31.62+330 \cdot \cos \theta$ & All & See above & $\begin{array}{l}\text { Reduce events caused by } \\
\text { mostly downward moving } \\
\text { air shower muons }\end{array}$ \\
\hline
\end{tabular}

Table 4 Description of all cuts in the mildly relativistic analysis and the according event rate

\begin{tabular}{|c|c|c|c|c|}
\hline Cut variable & Cut value & Data rate $[\mathrm{Hz}]$ & Description & Motivation \\
\hline$\theta$ & $\geq 86^{\circ}$ & $2.30 \times 10^{1}$ & $\begin{array}{l}\text { Reconstructed zenith angle using improved } \\
\text { LineFit }\end{array}$ & $\begin{array}{l}\text { Reduce muons from air showers which } \\
\text { are significantly reduced at this angle } \\
\text { because of the thick atmosphere and ice; } \\
\text { this also requires a cut on the successful } \\
\text { fit-status of the reconstruction }\end{array}$ \\
\hline$v$ & $\leq 0.83 \quad \mathrm{c}$ & & Reconstructed velocity & $\begin{array}{l}\text { Only used in training to focus on low } \\
\text { velocities }\end{array}$ \\
\hline$n_{\text {String }}$ & $\geq 2$ & $1.86 \times 10^{1}$ & Number of hit strings & $\begin{array}{l}\text { Improve data quality and reduce pure } \\
\text { noise events }\end{array}$ \\
\hline$n_{\mathrm{DOM}}$ & $\geq 6$ & $1.64 \times 10^{1}$ & Number of hit DOMs & $\begin{array}{l}\text { Improve data quality and reduce pure } \\
\text { noise events }\end{array}$ \\
\hline$d_{\text {Gap } 100}$ & $\leq 300 \mathrm{~m}$ & $1.41 \times 10^{1}$ & $\begin{array}{l}\text { The maximal track length of the track, which got } \\
\text { no hits within the specified track cylinder } \\
\text { radius in meters }\end{array}$ & Reduce coincident events and noise events \\
\hline$d_{\text {Separation }}$ & $\geq 350 \mathrm{~m}$ & $2.62 \times 10^{-1}$ & $\begin{array}{l}\text { The distance the Center-of-Gravity (CoG) } \\
\text { positions of the first and the last quartile of the } \\
\text { hits, within the specified track cylinder radius, } \\
\text { are separated from each other }\end{array}$ & $\begin{array}{l}\text { Reduce down-going events, } \\
\text { corner-clippers, and cascades }\end{array}$ \\
\hline$z_{\mathrm{CoG}}$ & $\geq-400 \mathrm{~m}$ & $2.40 \times 10^{-1}$ & $\begin{array}{l}\text { The } \mathrm{z} \text { value of the position of the CoG of the } \\
\text { event }\end{array}$ & $\begin{array}{l}\text { Reduce horizontally mis-reconstructed } \\
\text { high energy tracks at the bottom of the } \\
\text { detector }\end{array}$ \\
\hline$z_{\mathrm{DOM}}$ & & & height $z$ of the position of a certain DOM & \\
\hline$z_{\text {travel }}$ & $\geq 0 \mathrm{~m}$ & $1.30 \times 10^{-1}$ & $\begin{array}{l}\text { Average penetration depth of hits defined from } \\
\text { below: The average over ( } z_{\text {DOM }} \text { minus the } \\
\text { average over the } z_{\text {DOM values of the first }} \\
\text { quartile of all hits) }\end{array}$ & $\begin{array}{l}\text { Reduce coincident events, down-going } \\
\text { tracks and cascades }\end{array}$ \\
\hline BDT Score & $\geq 0.47$ & $1.12 \times 10^{-7}$ & $\begin{array}{l}\text { Score reaching from }-1 \text { to } 1 \text { representing how } \\
\text { signal-like an event is }\end{array}$ & $\begin{array}{l}\text { For the choice of the value see text; see } \\
\text { Table } 5 \text { for the used variables }\end{array}$ \\
\hline
\end{tabular}


Table 5 Description of the variables used in the BDTs of the mildly relativistic analysis

\begin{tabular}{|c|c|c|}
\hline mRMR importance & BDT variable & Description \\
\hline 1 & $n_{\text {DOM } 100}$ & $\begin{array}{l}\text { The number of hit DOMs within the specified cylinder radius in meters around the } \\
\text { reconstructed track }\end{array}$ \\
\hline 2 & $\bar{s}$ & The mean of all distances of hits from the reconstructed track \\
\hline 3 & $t_{\mathrm{Gap}}$ & Largest time gap between all hits ordered by time \\
\hline 4 & $d_{\text {Gap } 100}$ & See above \\
\hline 5 & $d_{\text {Separation }}$ & See above \\
\hline 6 & $\bar{s}_{\mathrm{NPE}}$ & The average DOM distance from the track weighted by the total charge of each DOM \\
\hline 7 & $n_{\mathrm{DOM}}^{*} 50$ & $\begin{array}{l}\text { The number of DOMs with no hit within the specified cylinder radius in meters around } \\
\text { the reconstructed track }\end{array}$ \\
\hline 8 & $z_{\text {travel }}$ & See above \\
\hline 9 & $z_{\text {pattern }}$ & 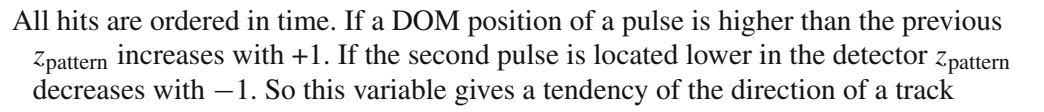 \\
\hline 10 & $n_{\text {DOM } 50}$ & $\begin{array}{l}\text { The number of hit DOMs within the specified cylinder radius in meters around the } \\
\text { reconstructed track }\end{array}$ \\
\hline 11 & $v$ & See above \\
\hline 12 & $k_{100}$ & $\begin{array}{l}\text { The smoothness values reaching from }-1 \text { to } 1 \text { how smooth the hits are distributed within } \\
\text { the specified cylinder radius around the reconstructed track }\end{array}$ \\
\hline 13 & $t_{w}$ & $\begin{array}{l}\text { The weighted deviation of all hit times from the charge weighted mean of all hit times } \\
\text { distribution }\end{array}$ \\
\hline 14 & $t$ & $\begin{array}{l}\text { Time length of an event; calculated by ordering all hits in time and subtracting the last } \\
\text { minus the first time value }\end{array}$ \\
\hline 15 & $\bar{z}_{\mathrm{DOM}}$ & Mean of all $z_{\text {DOM }}$ per event \\
\hline
\end{tabular}

\section{References}

1. G. 't Hooft, Nucl. Phys. B 79, 276 (1974)

2. A.M. Polyakov, JETP Lett. 20, 194 (1974)

3. A.H. Guth, S.H.H. Tye, Phys. Rev. Lett. 44(10), 631 (1980)

4. J. Polchinski, Int. J. Mod. Phys. A 19, 145 (2004). doi:10.1142/ S0217751X0401866X

5. P. Dirac, Proc. R. Soc. A 133, 60 (1931)

6. J.P. Preskill, Ann. Rev. Nucl. Part. Sci. 34, 461 (1984)

7. S.D. Wick, T.W. Kephart, T.J. Weiler, P.L. Biermann, Astropart. Phys. 18(6), 663 (2003)

8. S. Dar, Q. Shafi, A. Sil, Phys. Rev. D 74, 035013 (2006)

9. M. Sakellariadou, Lect. Notes Phys. 738, 359 (2008)

10. A. Achterberg et al., Astropart. Phys. 26, 155 (2006). doi:10.1016/ j.astropartphys.2006.06.007

11. R. Abbasi et al., Nucl. Instrum. Methods A 700, 188 (2014)

12. R. Abbasi et al., Nucl. Instrum. Methods A 618(1-3), 139 (2010)

13. R. Abbasi et al., Astropart. Phys. 35(10), 615 (2012)

14. M. Ackermann, et al., J. Geophys. Res. 111(D13) (2006)

15. M.G. Aartsen, et al., Nucl. Instrum. Methods A 711, 73 (2013)

16. R. Abbasi et al., Nucl. Instrum. Methods A 601(3), 2994 (2009)

17. M.G. Aartsen, et al., Nucl. Instrum. Methods A 736, 143 (2014)

18. A. Roodman, in Proceedings of the conference on Statistical Problems in Particle Physics, Astrophysics, and Cosmology (2003), p. 166. arXiv:physics/0312102

19. S. Adrián-Martínez, et al., Astropart. Phys. 35, 634 (2012). doi:10. 1016/j.astropartphys.2012.02.007

20. F. Moulin, Il Nuovo Cimento B 116, 869 (2001)

21. E. Tamm, M. Frank, Dokl. Akad. Nauk SSSR (Akad. of Science of the USSR), 14, 107 (1937)

22. D.R. Tompkins, Phys. Rev. 138(1B) (1964)

23. T.T. Wu, C.N. Yang, Nucl. Phys. B 107, 365 (1976)
24. Y. Kazama, C.N. Yang, A.S. Goldhaber, Phys. Rev. D 15, 2287 (1977)

25. E. Bauer, Math. Proc. Camb. Philos. Soc. 47(04), 777 (1951). doi:10.1017/S0305004100027225

26. H.J.D. Cole, Math. Proc. Camb. Philos. Soc. 47(01), 196 (1951)

27. S.P. Ahlen, Phys. Rev. D 14, 2935 (1975)

28. S.P. Ahlen, Phys. Rev. D 17(1), 229 (1978)

29. R.M. Sternheimer, At. Data Nucl. Data Tables 30(2), 261 (1984)

30. L.I. Grossweiner, M.S. Matheson, J. Chem. Phys. 20(10), 1654 (1952). doi:10.1063/1.1700246

31. L.I. Grossweiner, M.S. Matheson, J. Chem. Phys. 22(9), 1514 (1954). doi:10.1063/1.1740451

32. T.I. Quickenden, S.M. Trotman, D.F. Sangster, J. Chem. Phys. 77, 3790 (1982). doi:10.1063/1.444352

33. V. Aynutdinov et al., Astrophys. J. 29, 366 (2008)

34. J.R. Hoerandel, Astropart. Phys. 19(2), 193 (2003). doi:10.1016/ S0927-6505(02)00198-6

35. T.K. Gaisser, Astropart. Phys. 35(12), 801 (2012)

36. M. Honda, T. Kajita, K. Kasahara, S. Midorikawa, T. Sanuki, Phys. Rev. D 75(4), 043006 (2007)

37. R. Enberg, M.H. Reno, I. Sarcevic, Phys. Rev. D 78(4), 043005 (2008)

38. M.G. Aartsen, et al., Science 342(6161) (2013). doi:10.1126/ science. 1242856

39. M.G. Aartsen et al., Phys. Rev. Lett. 113(10), 101101 (2014)

40. J. Lundberg et al., Nucl. Instrum. Methods A 581, 619 (2007)

41. R. Abbasi, et al., Phys. Rev. D 87, 022001 (2013)

42. J. Posselt, Search for Relativistic Magnetic Monopoles with the IceCube 40-String Detector. Ph.D. thesis, University of Wuppertal (2013)

43. Y. Freund, Inform. Comput. 121(2), 256 (1995). doi:10.1006/inco. 1995.1136 
44. H. Peng, I.E.E.E. Trans, Pattern Anal. Mach. Intell. 27(8), 1226 (2005). doi:10.1109/TPAMI.2005.159

45. B. Efron, Ann. Stat. 7(1), 1 (1979)

46. J. Kunnen, J. Luenemann, A. Obertacke Pollmann, F. Scheriau for the IceCube Collaboration, in proceedings of the 34th International Cosmic Ray Conference (2015), p. 361. arXiv: 1510.05226

47. G.J. Feldman, R.D. Cousins, Phys. Rev. D 57(7), 3873 (1998)

48. M. Ambrosio et al., Eur. Phys. J. C 25, 511 (2002)

49. E.N. Parker, Astrophys. J. 160, 383 (1970)

50. W. Haerdle, Z. Hlavka, Multivariate Statistics (Springer New York, 2007). doi:10.1007/978-0-387-73508-5

51. R. Abbasi et al., Eur. Phys. J. C 69, 361 (2010)

52. M.G. Aartsen et al., Eur. Phys. J. C 74, 2938 (2014)
53. Y.F. Novoseltsev, M.M. Boliev, A.V. Butkevich, S.P. Mikheev, V.B. Petkov, Nucl. Phys. B, Proc. Suppl. 151, 337 (2006). doi:DOIurl10.1016/j.nuclphysbps.2005.07.048

54. A. Pollmann, Search for mildly relativistic Magnetic Monopoles with IceCube. Ph.D. thesis, University of Wuppertal (Submitted)

55. S. Adrian-Martinez, et al. The prototype detection unit of the KM3NeT detector (2014). arXiv: 1510.01561

56. I.E. Bojaddaini, G.E. Pavalas, in Proceedings of the 34th International Cosmic Ray Conference (2015), p. 1097

57. M.G. Aartsen, et al., IceCube-Gen2: a vision for the future of neutrino astronomy in Antarctica (2014). arXiv:1412.5106

58. M.G. Aartsen, et al., Letter of intent: the precision icecube next generation upgrade (PINGU) (2014). arXiv:1401.2046 